\title{
Benzocaína e eugenol como anestésicos para juvenis do pampo Trachinotus marginatus
}

\author{
Benzocaine and eugenol as anaesthetics for pompano juvenile Trachinotus marginatus
}

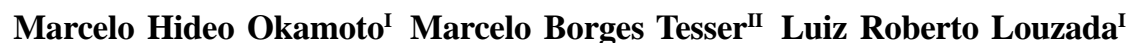 \\ Renato Adriano dos Santos ${ }^{\mathrm{III}}$ Luís André Sampaio ${ }^{\mathrm{II}}{ }^{*}$
}

\section{RESUMO}

O objetivo deste trabalho foi avaliar a eficácia da benzocaína e do eugenol como anestésicos para juvenis de Trachinotus marginatus. Foram testadas concentrações de 25, 50, 75, 100 e 150ppm de benzocaína e de 25, 37,5, 50, 62,5 e $75 \mathrm{ppm}$ de eugenol. Os testes foram realizados em aquários de vidro contendo 301 de água (salinidade 30\% e temperatura $\left.19^{\circ} \mathrm{C}\right)$. Os peixes $(51,4 \pm 13,5 \mathrm{~g})$ foram expostos individualmente às diferentes concentrações de cada anestésico ( $n=5$ para cada concentração). Enquanto ainda anestesiados, eles foram medidos e pesados para posteriormente serem colocados em um aquário sem anestésico para recuperação. Posteriormente, os peixes foram colocados em um aquário sem anestésico para observação da recuperação. O tratamento estatístico dos resultados foi feito pela Análise de Variância (One-Way - ANOVA) com 95\% de significância e, quando encontradas diferenças significativas, o teste de Duncan foi aplicado. Os períodos para atingir a latência para os juvenis de pampos variaram entre 11 min para a concentração de $25 \mathrm{ppm}$ e $2 \mathrm{~min}$ na concentração de 150 ppm para a benzocaína e de 10min (25ppm) a $2 \mathrm{~min}$ (75ppm) para o eugenol. Os tempos de recuperação foram entre 3 min para 25 ppm e 14 min para a concentração de 150 ppm para a benzocaína e de $2 \mathrm{~min}$ (25ppm) a 8min (75ppm) para o eugenol. A dose de 50ppm de benzocaína e eugenol proporcionou um período de latência e recuperação dentro da faixa considerada adequada (3mim para a latência e $5 \mathrm{mim}$ para a recuperação). Entretanto, é importante salientar que o custo da benzocaína para anestesiar juvenis de pampo é 1/3 inferior ao custo do eugenol.

Palavras-chave: anestesia, manejo, piscicultura marinha, Trachinotus marginatus.

\begin{abstract}
The aim of this experiment was to evaluate the efficacy of benzocaine and eugenol as anesthetics for Trachinotus marginatus juveniles. Five concentrations of benzocaine $(25,50,75,100$, and 150ppm) and eugenol (25, $37.5,50,62.5$, and $75 \mathrm{ppm}$ ) were tested. The trials were conducted in glass aquariums filled with 301 seawater (salinity $30 \%$ and temperature $19^{\circ} \mathrm{C}$ ). The fish $(51.4 \pm 13.5 \mathrm{~g})$ were individually exposed to different concentrations of each anesthetic ( $n=5$ for each concentration). They were measured and weighed under anesthesia, and then returned to another aquarium without anesthetic for recovery. The results were evaluated using Analyses of Variance (One-Way - ANOVA) followed by the test of Duncan (95\% confidence level) when necessary. Anesthesia time varied from approximately $11 \mathrm{~min}$ (25ppm) to $2 \mathrm{~min}$ (150ppm) for benzocaine, and from 10min (25ppm) to $2 \mathrm{~min}$ (75ppm) for eugenol. Recovery time ranged from 3min (25ppm) to $14 \mathrm{~min}$ (150ppm) for benzocaine and from $2 \mathrm{~min}$ (25ppm) to $8 \mathrm{~min}$ (75ppm) for eugenol. Benzocaine and eugenol, both at 50ppm, induce quick anesthesia and recovery (3mim for anesthesia and $5 \mathrm{mim}$ for recovery) for juvenile Trachinotus marginatus, and therefore are considered efficient anesthetics for this species. However, it is important to emphasize that the cost of benzocaine is $1 / 3$ lower than eugenol to anesthetize juvenile pompano.
\end{abstract}

Key words: anesthesia, handling, marine fish culture, Trachinotus marginatus.

\section{INTRODUÇÃO}

Durante práticas como biometria, transporte, extrusão de gametas e marcação, é importante anestesiar

IPrograma de Pós-graduação em Aqüicultura, Instituto de Oceanografia, Universidade Federal do Rio Grande (FURG), Rio Grande, RS, Brasil.

IILaboratório de Maricultura, Instituto de Oceanografia, FURG, Rio Grande, RS, Brasil. E-mail: sampaio@mikrus.com.br. *Autor para correspondência.

IIICurso de Oceanologia, FURG, Rio Grande, RS, Brasil. 
os peixes com o objetivo de facilitar o manejo e reduzir o estresse e a possibilidade de ocorrer injúrias (Ross \& Ross, 1999; Maršic-LUCIC et al., 2005). A escolha de um anestésico deve ser baseada em critérios como a eficácia, o custo, a disponibilidade no mercado, a segurança durante o uso e os possíveis efeitos colaterais aos peixes, aos seres humanos e ao meio ambiente (MARKING \& MEYER, 1985).

Diversos produtos químicos são utilizados para a anestesia de peixes, sendo os mais comuns a tricaína metanosulfato (MS-222), a quinaldina e o 2fenoxietanol (MGBENKA \& EJIOFOR, 1998; ROSS \& ROSS, 1999; HOVDA \& LINLEY, 2000). No Brasil, o anestésico mais utilizado é a benzocaína (GOMES et al., 2001). Esse anestésico atende a maioria dos critérios estabelecidos por ROSS \& ROSS (1999), além de ter baixo custo e boa margem de segurança para os peixes (GILDERHUS \& MARKING, 1987; GILDERHUS, 1989). Outro anestésico bastante utilizado é o eugenol (ROUBACH et al., 2005). Este é um produto de origem natural, derivado do óleo de cravo (ISAACS, 1983). Além de apresentar ampla disponibilidade no mercado e baixo custo, o eugenol é considerado seguro para o meio ambiente e para o manipulador (IVERSEN et al., 2003).

O pampo Trachinotus marginatus é uma espécie endêmica do Oceano Atlântico Sul Ocidental, ocorrendo desde o Estado do Rio de Janeiro até o Norte da Argentina em águas costeiras e estuarinas (FISCHER et al., 2004). Espécies do gênero Trachinotus vêm sendo consideradas para a piscicultura em função da rápida adaptação ao cativeiro, da boa tolerância a condições ambientais extremas e do rápido crescimento (JORY et al., 1985; SAMPAIO et al., 2003). Porém, informações elementares a respeito do seu manejo são ainda necessárias. Dessa forma, o objetivo deste trabalho foi avaliar a eficácia dos anestésicos benzocaína e eugenol em juvenis do pampo T. marginatus.

\section{MATERIAL E MÉTODOS}

Juvenis de pampo foram capturados na Praia do Cassino, em Rio Grande, Rio Grande do Sul (RS), no mês de fevereiro de 2005, com rede de arrasto. Os animais foram mantidos por seis meses em um tanque contendo 1.0001 de água do mar (salinidade 30\%o). Durante esse período, os peixes foram alimentados com ração comercial (NRD INVE, 59\% proteína). Os testes foram realizados em aquários de vidro contendo 301 de água (salinidade $30 \%$ e temperatura $19^{\circ} \mathrm{C}$ ). Um aquário foi utilizado para a indução à anestesia e o outro para a observação da recuperação. O preparo das soluções estoque foi feito com a diluição de 1:9 (p:v) de benzocaína (Henrifarma Produtos Químicos e Farmacêuticos LTDA) em álcool e 1:2 (v:v) de eugenol (Asfer Indústria Química LTDA) com álcool comercial. Os peixes $(51,4 \pm 13,5 \mathrm{~g}$, média $\pm \mathrm{DP})$ foram retirados aleatoriamente do tanque por meio de puçás e expostos individualmente às diferentes concentrações de cada anestésico ( $\mathrm{n}=5$ para cada concentração). De acordo com MUNDAY \& WILSON (1997), a concentração de álcool, utilizado como diluente para benzocaína e eugenol, não é suficiente para influenciar o padrão de anestesia.

Inicialmente foi examinado o efeito das diferentes concentrações de benzocaína e eugenol sobre o tempo necessário para a latência total dos peixes, considerado o período entre o início da exposição até a perda total de equilíbrio na coluna de água e a parada dos batimentos operculares, condição equivalente ao estágio III de anestesia de acordo com IWAMA et al. (1989). Após atingir o estágio III, os peixes foram medidos (comprimento total), pesados e transferidos, com puçás, para um aquário com água sem anestésico, onde foi monitorado o tempo de recuperação, considerado como o período necessário para que os peixes recuperassem o equilíbrio e a natação ativa na coluna de água, equivalente ao estágio III de recuperação de IWAMA et al. (1989). No momento em que os peixes foram colocados nos aquários, a mangueira de aeração foi retirada para facilitar a visualização. Após os testes de anestesia e recuperação, os animais ficaram em observação durante 24h em um tanque contendo $100 \mathrm{l}$ de água para a avaliação da sobrevivência.

O tempo de anestesia, bem como o tempo de recuperação, foi obtido por meio de um cronômetro digital. A água dos aquários foi totalmente trocada a cada teste. Foram testadas as concentrações 25, 50, 75, 100 e 150ppm para a benzocaína e 25, 37,5, 50, 62,5 e $75 \mathrm{ppm}$ para o eugenol. Os testes com cada anestésico foram realizados individualmente e em dias separados. Os testes foram realizados sempre da menor para a maior concentração, e os aquários foram lavados com água do mar antes de se avaliar uma nova concentração.

Os resultados foram analisados por meio da Análise de Variância (One-Way - ANOVA) e quando encontradas diferenças significativas foi aplicado o Teste de Duncan. Todas as análises foram realizadas ao nível de significância de 95\%.

\section{RESULTADOS}

Os resultados sobre o tempo de anestesia e recuperação dos juvenis de pampo expostos às diferentes concentrações de benzocaína e eugenol 
encontram-se nas figuras 1 e 2, respectivamente. Todos os peixes atingiram o estágio III de latência, tanto para a benzocaína, quanto para o eugenol em todas as concentrações testadas. Não houve mortalidade durante ou após os testes.

Os peixes submetidos às diferentes concentrações de benzocaína atingiram o estágio III de latência em três faixas de tempo distintas. A concentração de 25ppm resultou em maior tempo para atingir a latência (aproximadamente $11 \mathrm{~min}$ ), enquanto nas concentrações de 100 e 150ppm o tempo para a latência foi significativamente $(\mathrm{P}<0,05)$ mais rápido (aproximadamente 2min). Um intervalo de tempo intermediário (aproximadamente 3min) foi observado quando foram testadas as concentrações de 50 e 75ppm de benzocaína (Figura 1A). O tempo de recuperação teve uma relação dose-dependente variando de $2 \mathrm{~min}$ na concentração de $25 \mathrm{ppm}$ a $13 \mathrm{~min}$ para a maior concentração de benzocaína avaliada (150ppm) (Figura 1B).

Assim como ocorreu com a benzocaína, os peixes submetidos à concentração mais baixa de eugenol (25ppm) levaram mais tempo para atingir a latência total (aproximadamente 10min) do que na maior concentração (75ppm), cujo tempo foi de $2 \mathrm{~min}$ (Figura 2A). O tempo de recuperação mostrou um padrão inverso ao tempo de anestesia, variando aproximadamente de 2 min na concentração de 25ppm a 8min quando os pampos foram expostos na concentração de 75ppm de eugenol (Figura 2B).

A partir das diluições descritas para a produção das soluções estoques de eugenol e benzocaína e a concentração ideal para anestesia de pampos, foi calculado o custo de uma anestesia em tanques de 100l. Para tanto, foram utilizados os valores de R \$ 4,00 o litro de álcool, R\$ 103,00 o litro de eugenol e R\$ 62,00 o kg de benzocaína, valores obtidos junto às empresas fornecedoras dos produtos. Com a concentração de 50ppm de cada anestésico, o custo para anestesiar juvenis de pampo em tanques de 1001 foi de R \$ 0,50 para benzocaína e R \$1,60 para o eugenol.

\section{DISCUSSÃO}

MARKING \& MEYER (1985) sugeriram que a eficiência de um anestésico está no curto tempo de latência (aproximadamente $3 \mathrm{~min}$ ) e na rápida recuperação dos peixes (aproximadamente $5 \mathrm{~min}$ ). Segundo tal recomendação, os dois produtos testados mostraram-se eficazes, sendo que a concentração considerada ideal para a benzocaína foi de 50ppm. A concentração de 50ppm também foi considerada ideal para o eugenol. Apesar do tempo de latência também ser curto nas concentrações de 62,5 e 75ppm, o tempo de recuperação nessas duas concentrações foi considerado elevado.

Outra característica a ser observada na escolha do anestésico é a sua segurança para os peixes a fim de que não cause efeitos deletérios subletais. Anestésicos como o MS-222 e a quinaldina podem induzir problemas olfatórios em peixes (LOSEY \& HUGIE, 1994). Para juvenis de Menidia estor, ROSS et al. (2007) encontraram que concentrações acima de $40 \mathrm{mg} \mathrm{L}^{-1}$ de benzocaína resultam em hemorragia nas nadadeiras e na cabeça, que levaram posteriormente os peixes à morte.

SLADKY et al. (2001) observaram que pacus submetidos à anestesia com eugenol nas concentrações

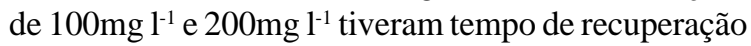
acima de $10 \mathrm{~min}$ e alguns foram reanimados com auxílio de oxigenação extra perto da cavidade opercular. Valores próximos aos 10min de anestesia foram encontrados
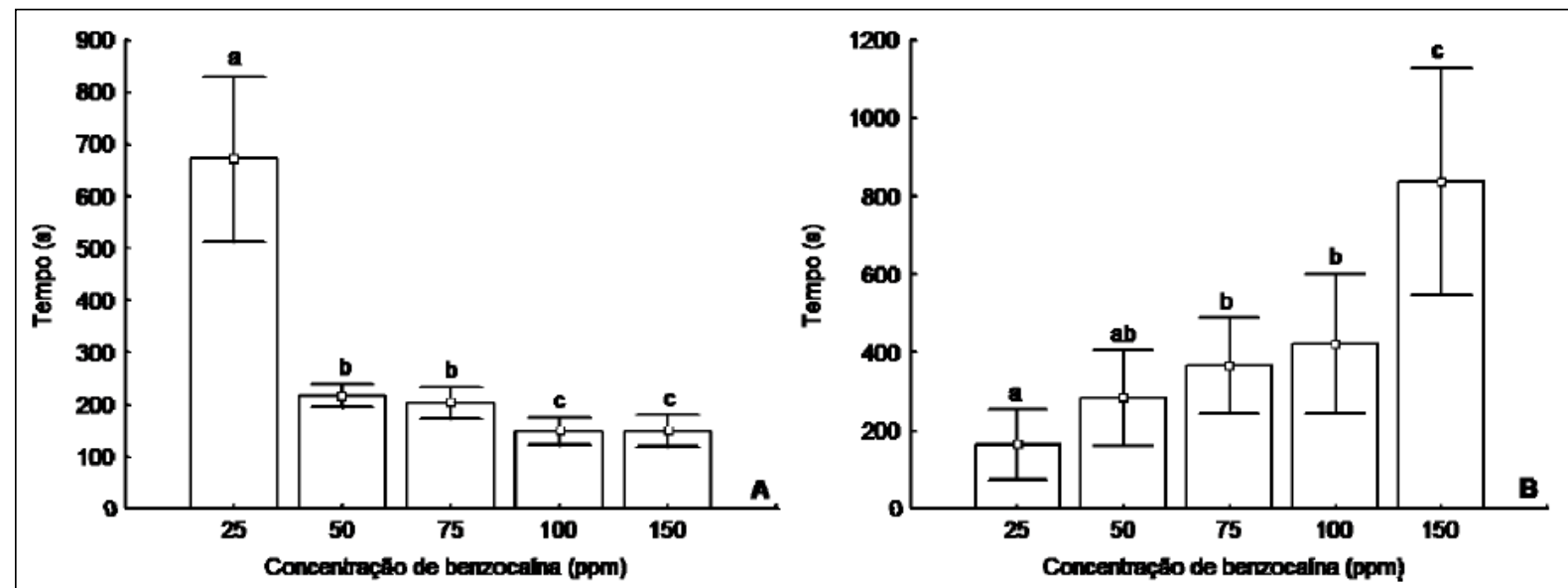

Figura 1 - Tempo (média \pm DP) em que juvenis de Trachinotus marginatus atingiram o período de latência (A) e a recuperação (B) com o uso de benzocaína. Letras diferentes indicam diferença significativa $(\mathrm{P}<0,05)$. 

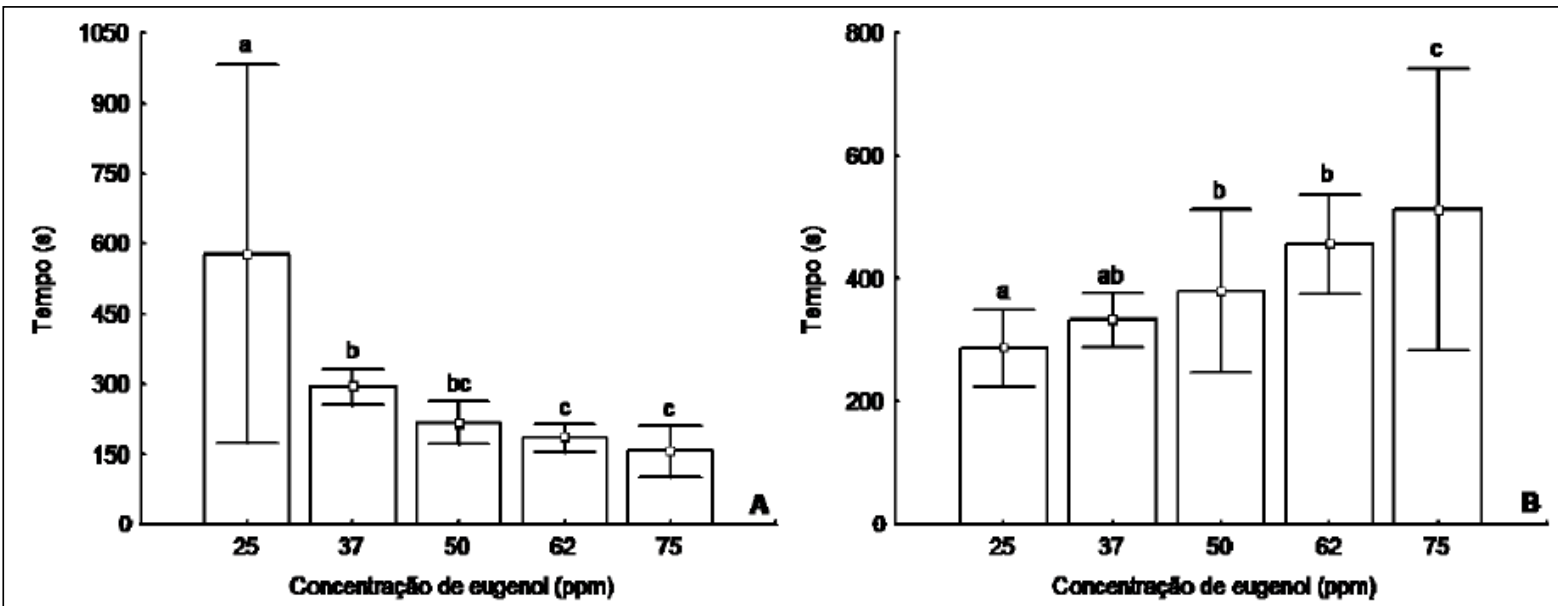

Figura 2 - Tempo (média \pm DP) em que juvenis de Trachinotus marginatus atingiram o período de latência (A) e a recuperação (B) com o uso de eugenol. Letras diferentes indicam diferença significativa $(\mathrm{P}<0,05)$.

para os pampos submetidos à concentração de 75ppm, mas não houve necessidade de auxílio para o retorno da anestesia. Esse fato corrobora o fato que o eugenol possui uma margem de segurança reduzida quando comparado com outras substâncias. Não houve mortalidade durante ou após os testes dentro das concentrações testadas. No entanto, novos estudos devem ser conduzidos com o objetivo de se verificar a influência desses anestésicos sobre os efeitos subletais para juvenis de pampo.

ALLEN (1988) determinou que a benzocaína não foi detectada no músculo dos peixes anestesiados 24 horas após seu uso, período considerado curto se comparado com MS-222, que pode ser detectado até 21 dias após a anestesia (WOOD et al., 2002). Nesse aspecto, o eugenol possui vantagem sobre a benzocaína por ser rapidamente metabolizado e excretado, não havendo necessidade de depuração (CHO \& HEATH, 2000). No entanto, estudos recentes revelam que a administração sucessiva de eugenol pode levar à acumulação do produto em trutas e que novos estudos devem ser conduzidos para determinar sua toxicidade (GUENETTE et al., 2007).

A segurança das pessoas durante a manipulação de tais substâncias deve ser levada em consideração para a escolha do anestésico. FAÇANHA \& GOMES (2005) classificaram a benzocaína e o eugenol como substâncias seguras para serem manipuladas, no entanto, LI et al. (2008) demonstraram que a benzocaína pode causar eczema em 2,3\% da população amostral de seres humanos. FENG \& LIPTON (1998) apontam que o eugenol é irritante quando aplicado topicamente em ratos de laboratórios, coelhos e cães, causando inflamação local e necrose celular.

Os dois produtos testados são facilmente encontrados no mercado e possuem preços acessíveis, ao contrário do MS-222, que não é produzido no país e chega a custar 10 vezes mais que a benzocaína para atingir uma concentração necessária para anestesiar peixes (GOMES et al., 2001). A partir dos valores de mercado e das condições experimentais deste trabalho foi determinado que o custo para induzir a latência nos pampos com eugenol é cerca de três vezes superior do que com benzocaína.

\section{CONCLUSÕES}

O curto período de anestesia e recuperação na concentração de 50ppm de benzocaína e de eugenol qualificam tais substâncias como bons anestésicos para juvenis do pampo T. marginatus. No entanto, em termos econômicos, a benzocaína apresenta melhor custo/benefício.

\section{AGRADECIMENTOS}

Os autores agradecem à Coordenação de Aperfeiçoamento de Pessoal de Nível Superior (CAPES), pela Bolsa de Doutorado para M.H. Okamoto e ao Conselho Nacional de Desenvolvimento Científico e Tecnológico (CNPq), pela Bolsa de Iniciação Científica (PIBIC CNPq-FURG) para R.A. dos Santos e pela Bolsa de Produtividade em Pesquisa para L.A. Sampaio (Processo 301673/2006-3).

\section{REFERÊNCIAS}

ALLEN, J.L. Residues of benzocaine in rainbow trout, largemouth bass, and fish meal. Progressive Fish-Culturist, Bethesda, v.50, n.1, p.59-60, 1988.

CHO, G.K; HEATH, D.D. Comparison of tricaine methanesulphonate (MS 222) and clove oil anesthesia effects on the physiology of juvenile chinook salmon Oncorhynchus tshawytscha Walbaum). Aquaculture Research, v.31, p.537546, 2000

Ciência Rural, v.39, n.3, mai-jun, 2009. 
FAÇANHA, M.F.; GOMES, L.C. A eficácia do mentol como anestésico para o tambaqui (Colossoma macropomum, Characiformes: Characidae). Acta Amazonica, Manaus, v.35, n.1, p.71-75, 2005.

FENG J.; LIPTON, J.M. Eugenol: antipyretic activity in rabbits. Neuropharmacology, Oxford, v.26, p.1775-1778, 1998.

FISCHER, L.C. et al. Peixes estuarinos e costeiros. Rio Grande: Ecoscientia, 2004. 127p.

GILDERHUS, P.A. Efficacy of benzocaine as an anesthetic for salmonid fishes. North American Journal of Fisheries Management, Bethesda, v.9, p.150-153, 1989.

GILDERHUS, P.A.; MARKING, L.L. Comparative efficacy of 16 anesthetic chemicals on rainbow trout. North American Journal of Fisheries Management, Bethesda, v.7, p.288292, 1987.

GOMES, L.C. et al. Efficacy of benzocaine as an anesthetic in juvenile tambaqui Colossoma macropomum. Journal of the World Aquaculture Society, Baton Rouge, v.32, n.4, p.426431, 2001.

GUENETTE, S.A. et al. Pharmacokinetics of eugenol in rainbow trout (Oncorhynchus mykiss). Aquaculture, Amsterdam, v.266, n.1-4, p.262-265, 2007.

HOVDA, J.; LINLEY, T.J. The potential application of hypothermia for anesthesia in adult Pacific salmon. North American Journal of Aquaculture, Bethesda, v.62, p.6772, 2000.

ISAACS, G. Permanent local anaesthesia and anhidrosis after clove oil spillage. Lancet, Oxford, v.321, p.882, 1983.

IVERSEN, M. et al. The efficacy of metomidate, clove oil, AQUI-S ${ }^{\mathrm{TM}}$ and Benzoak ${ }^{\circledR}$ as anaesthetics in Atlantic salmon (Salmo salar L.) smolts, and their potential stress-reducing capacity. Aquaculture, Amsterdam, v.221, p.549-566, 2003.

IWAMA, G.K. et al. The effects of five fish anesthetics on acid-base balance, hematocrit, blood gases, cortisol, and adrenaline in rainbow trout. Canadian Journal of Zoology, Toronto, v.67, p.2065-2073, 1989.

JORY, D. et al. Culture of the fishes of the genus Trachinotus (Carangidae) in the Western Atlantic. Journal of the World Mariculture Society, Baton Rouge, v.16, p.87-94, 1985.
LI, L-F. et al. Etiology and prognosis of hand eczema in a dermatology clinic in China: a follow-up study. Contact Dermatitis, Oxon, v. 58, p. 88-92, 2008.

LOSEY, G.S.; HUGIE, D.M. Prior anaesthesia impairs a chemically mediated fright response in a gobiid fish. Journal of Chemical Ecology, Amsterdam, v.20, n.8, p.1877-1883, 1994.

MARKING, L.L.; MEYER, F.P. ARE BETTER FISH ANESthetics needed in fisheries? Fisheries, Bethesda, v.10, n.6, p.2-5, 1985 .

MARŠIC-LUCIC, J. et al. Comparative effectiveness of 2phenoxyethanol and propiscin as anesthetics for juvenile sea bass Dicentrarchus labrax L. Aquaculture International, Dordrecht, v.13, p.543-553, 2005.

MGBENKA, B.O.; EJIOFOR, E.N. Effects of extracts of dried leaves of Erythrophleum suaveolens as anesthetics on clariid catfish. Journal of Applied Aquaculture, Binghamton, v.8, p.73-80, 1998.

MUNDAY P.L.; WILSON S.K. Comparative efficacy of clove oil and other chemicals in anaesthetization of Pomacentrus amboinensis, a coral reef fish. Journal of Fish Biology, Oxford, v.51, p.931-938, 1997.

ROSS, L.G. et al. Anesthesia, sedation and transportation of juvenile Menidia estor (Jordan) using benzocaine and hypothermia. Aquaculture Research, Oxon, v.38, p.909917, 2007.

ROSS, L.G.; ROSS, B. Anaesthetic and sedative techniques for aquatic animals. Oxford: Blackwell Science, 1999. 159p.

ROUBACH, R. et al. Eugenol as an efficacious anaesthetic for tambaqui Colossoma macropomum (Cuvier). Aquaculture Research, Oxon, v.36, n.11, p.1056-1061, 2005.

SAMPAIO, L.A. et al. Tolerância de juvenis do pampo Trachinotus marginatus (Teleostei,Carangidae) ao choque agudo de salinidade em laboratório. Ciência Rural, Santa Maria, v.33, n.4, p.757-761, 2003.

SLADKY, K.K. et al. Comparative efficacy of tricaine methanesulfonate and clove oil for use as anesthetics in red pacu (Piaractus brachypomus). American Journal of Veterinary Research, Davis, v.62, n.3, p.337-342, 2001.

WOOD, C.A. et al. Clove oil as an anaesthetic for adult sockeye salmon: field trials. Journal of Fish Biology, Oxford, v.60, p.340-347, 2002. 